\title{
Erratum to: Medical Image Computing and Computer-Assisted Intervention - MICCAI 2016
}

\author{
Sebastien Ourselin, Leo Joskowicz, Mert R. Sabuncu, Gozde Unal, \\ and William Wells
}

\section{Erratum to: \\ S. Ourselin et al. (Eds.): Medical Image Computing and Computer-Assisted Intervention - MICCAI 2016, LNCS 9902, https://doi.org/10.1007/978-3-319-46726-9}

The original version of the book was revised; the following corrections have been incorporated:

In Chapter "Anatomically Constrained Video-CT Registration via the V-IMLOP Algorithm":

The acknowledgement text of the initially published paper was missing. It should read as follows:

This work was funded by NIH R01-EB015530: Enhanced Navigation for Endoscopic Sinus Surgery through Video Analysis and NSF Graduate Research Fellowship Program.

In Chapter "Identifying Patients at Risk for Aortic Stenosis Through Learning from Multimodal Data":

The original version of this chapter was inadvertently published with incorrect author name "Yanrong Guo". This should be changed to "Yufan Guo". The correction to this chapter has been updated with the change.

The updated version of these chapters can be found at https://doi.org/10.1007/978-3-319-46726-9_16

https://doi.org/10.1007/978-3-319-46726-9_28

https://doi.org/10.1007/978-3-319-46726-9 